\title{
Uma Revisão Sistemática sobre a Educação do Surdo em Ambientes Virtuais Educacionais
}

\author{
Denys F. S. Rocha ${ }^{1}$, Ig Ibert Bittencourt ${ }^{1}$, Diego Dermeval ${ }^{2}$, Seiji Isotani ${ }^{3}$ \\ ${ }^{1}$ Instituto de Computação - Universidade Federal de Alagoas (UFAL) - Maceió - AL - \\ Brasil \\ ${ }^{2}$ Departamento de Sistemas e Computação - Universidade Federal de Campina Grande \\ (UFCG) - Campina Grande - PB - Brasil \\ ${ }^{3}$ Instituto de Ciências Matemáticas e de Computação - Universidade de São Paulo \\ (USP) - São Carlos - SP - Brasil
}

\begin{abstract}
Promoting Accessibility and Deaf Education is a topic much discussed in the academy and society in general. Nevertheless, there are few studies that describe the types of research and the most investigated topics in this area. Thus, a systematic review was conducted in order to get an overview of what has been done to promote the accessibility of deaf in virtual environments. Twenty-nine studies were selected at the end of the search and selection process. These studies were selected and ranked according to their contribution. As a final result of the studies a map was created showing where community efforts have focused in order to create assistive solutions for the education of the deaf in virtual environments as well as the techniques and approaches that have been used in this context.
\end{abstract}

Resumo. A promoção da Acessibilidade e Educação do Surdo é um tema bastante discutido na Academia e sociedade em geral. Apesar disso, há poucos estudos que retratam os tipos de pesquisas e os tópicos mais investigados nessa área. Dessa maneira, uma revisão sistemática foi realizada com o objetivo de obter uma visão geral do que tem sido feito para a promoção da acessibilidade do surdo em ambientes virtuais. Vinte e nove estudos foram selecionados ao final do processo de busca e seleção. Esses estudos foram selecionados e classificados de acordo com a sua contribuição. Como resultado final um mapa dos estudos foi criado mostrando onde os esforços da comunidade tem se concentrado com o intuito de criar soluções assistivas para a educação do surdo em ambientes virtuais, como também as técnicas e abordagens que tem sido utilizadas nesse contexto. 


\section{Introdução}

A WFD (World Federation of the Deaf - Federação Mundial dos Surdos) é uma organização internacional não governamental que representa aproximadamente 70 milhões de Surdos em todo o mundo. [Feneis 2014]. Segundo o último censo do Instituto Brasileiro de Geografia e Estatística (IBGE) realizado em 2010, a deficiência auditiva acometia 9,7 milhões de pessoas $(5,1 \%)$, das quais 344,2 mil eram surdas $(0,2 \%)$.

As pessoas surdas se comunicam através da Língua de Sinais. No Brasil temos a Língua Brasileira de Sinais (LIBRAS). Esta língua foi instituída através da lei $\mathrm{n}^{\mathbf{o}}$ 10.436, chamada de Lei de LIBRAS, onde a prática e o acesso à língua de sinais se tornam obrigatório em qualquer instituição onde houver um surdo. Mesmo com o apoio da legislação, o surdo ainda possui grande dificuldade de se inserir na sociedade. Por ser parte da minoria da população, sua forma de comunicação é ignorada, causando frustração e situações de desconforto. No cenário da Educação, não é diferente, Frias e Menezes (2008) confirmam essa visão de escola não inclusiva, dizendo que: "a escola historicamente se caracterizou pela visão da educação que delimita a escolarização como privilégio de alguns grupos, legitimando um processo de exclusão através de suas políticas e práticas educacionais, que reproduzem a ordem social."

Tecnologia Assistiva (TA) é um termo ainda novo, utilizado para identificar todo o arsenal de recursos e serviços que contribuem para proporcionar ou ampliar habilidades funcionais de pessoas com deficiência e consequentemente promover vida independente e inclusão [Bersch \& Tonolli apud Bersch 2013]. Tais tecnologias têm sido vistas como meio de promoção de inclusão social dentro da educação. Por este interesse, a comunidade acadêmica necessita de um amplo entendimento dos estudos existentes que apresentem uma visão geral sobre o apoio à educação dos surdos através das tecnologias assistivas. Com o objetivo de preencher esta necessidade, este trabalho realiza uma revisão sistemática das pesquisas existentes envolvendo surdo, tecnologias assistivas e ambientes virtuais. A revisão sistemática é uma metodologia que envolve a busca sistemática na literatura a fim de verificar a natureza, a extensão e a quantidade de estudos publicados (chamados de estudos primários) na área de interesse [Petersen et al. 2008].

O principal objetivo desta revisão é dar uma visão global do que está sendo pesquisado com relação à educação do surdo em ambientes virtuais nos últimos dez anos. Portanto, embora existam alguns artigos que propõem soluções utilizando algum tipo de tecnologia assistiva para suprir necessidades especiais (ex.: cegueira, deficiência intelectual, autismo e mobilidade reduzida), foram considerados somente estudos direcionados à necessidade da pessoa com surdez. Além disso, é importante ressaltar que esta revisão é parte de um trabalho de pesquisa mais amplo com o intuito de descobrir se há alguma contribuição direcionada ao modelo de educação inclusiva citado por Fonseca (2004), com foco na educação básica.

Neste trabalho são mostrados resultados de uma revisão sistemática, assim como seu processo de elaboração e desenvolvimento, desde o protocolo de pesquisa, até os resultados finais discutidos. A organização deste trabalho se dá da seguinte maneira: A Seção 2 descreve o método utilizado na realização da revisão sistemática. A Seção 3 apresenta e discute todos os resultados obtidos. A Seção 4 discute quais são as ameaças à validade do trabalho. Por fim, a Seção 6, as considerações finais deste trabalho. 


\section{Metodologia}

A Revisão Sistemática da Literatura (Systematic Literature Review - SLR) é um método empírico que tem por objetivos identificar, avaliar e interpretar questões de pesquisa, área de um tópico, ou fenômeno em uma pesquisa. Sua principal motivação é reunir provas para a fundamentação de conclusões [Kichenham e Charters 2007]. Kichenham e Charters (2007) indicam quais diretrizes são necessárias para a realização de uma SLR. De acordo com estas orientações, a SLR é dividida em algumas atividades, que podem ser agrupadas em três principais fases: (i) o planejamento da SLR, (ii) a realização da SLR, (iii) e relatório da SLR.

Para a realização da revisão, utilizou-se o modelo de protocolo da revisão sistemática descrita por Kichenham e Charters (2007). Este protocolo foi adaptado para abranger os requisitos desta revisão e os detalhes da condução, planejamento, avaliação e revisão são descritos nas subseções seguintes. Para apoiar a definição do protocolo desta SLR, foi utilizada a ferramenta START (State of the Art through Systematic Reviews).

O objetivo dessa revisão sistemática é recuperar e avaliar os estudos científicos que apresentam alguma contribuição na educação de surdos em ambientes virtuais. Dessa forma pretende-se responder à questão de pesquisa principal:

\section{O que tem sido feito pela educação do surdo em ambientes virtuais de aprendizagem?}

Com base na questão principal de pesquisa acima, outras perguntas mais específicas foram levantadas:

QP1 - Como é o processo de interação do surdo em ambientes virtuais?

QP2 - Quais as tecnologias assistivas utilizadas nos atuais ambientes virtuais de aprendizagem?

QP3 - Há alguma contribuição direcionada ao modelo de educação inclusiva, com foco na educação básica?

Após a definição das questões, o passo seguinte foi definir os critérios de seleção de busca. Esta revisão considera exclusivamente a busca automática, e foi validada por especialistas da área de informática na educação. A Figura 1 mostra o fluxo do processo de busca e seleção realizado nesta revisão.

Utilizando uma string de busca, a consulta automática foi realizada nas principais bases de dados de Ciência da Computação, sendo elas: ScienceDirect ${ }^{1}$, ISI Web of Science $^{2}$, Scopus ${ }^{3}$, SpringerLink ${ }^{4}$, ACM Digital Library ${ }^{5}$, IEEEXplore ${ }^{6}$ e Compendex $^{7}$, cobrindo dessa forma, os trabalhos mais relevantes da área. A Figura 1 mostra o processo de revisão sistemática e o número de artigos identificados em cada etapa. Dessa forma, a estratégia de busca e seleção (ou seja, a sequência de pesquisa e Passos 1-5) tem como objetivo capturar os estudos relacionados com os principais aspectos da área de tecnologias assistivas para pessoas surdas no contexto de educação.

\footnotetext{
${ }^{1}$ ScienceDirect

2 ISI Web of Science

${ }^{3} \underline{\text { Scopus }}$

${ }^{4}$ SpringerLink

5 ACM Digital Library

${ }^{6}$ IEEExplore

Compendex
} 
Os trabalhos selecionados devem ter como pré-requisito a existência de um ambiente virtual, podendo ter quaisquer técnicas computacionais envolvidas.

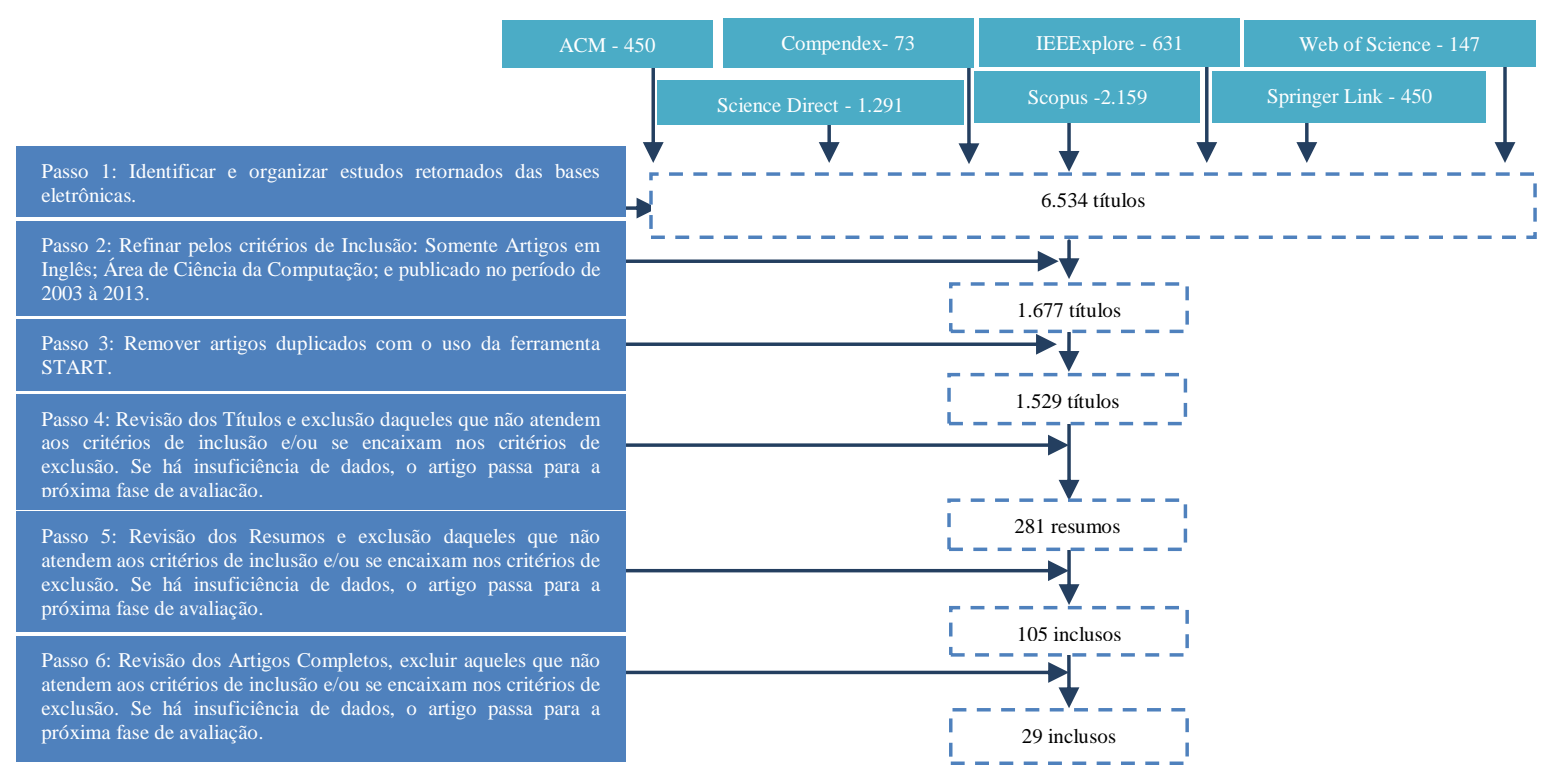

Figura 1. Fluxograma de seleção de artigos

Os estudos foram obtidos através de uma busca automática nas bases de dados eletrônicas através da utilização de alguns termos de pesquisa, onde foram agrupados em forma de uma "string de busca", descrita na Figura 2.

("deaf" OR "deafness" OR "hearing impairment" OR "sign language" OR "sign writing") AND ("online education" OR "inclusive education" OR "educational environments" OR "learning management systems" OR "online learning environment" OR "inclusive learning environment" OR "distance education" OR "e-learning" OR "m-learning" OR "t-learning" OR "collaborative learning" OR "computer supported collaborative learning" OR "cscl" OR "massive open online courses" OR "mooc" OR "intelligent tutoring system" OR "serious game" OR "assistive computing" OR "assistive technology")

\section{Figura 2. String de busca automática}

\subsection{Critérios de Inclusão e Exclusão}

O objetivo de definir um critério é identificar aqueles estudos primários que fornecem evidências diretas sobre as questões de pesquisa e também servem para reduzir a probabilidade de viés [Kitchenham and S. Charters 2007]. Os critérios de inclusão admitidos foram: Estudos da área de Ciência da Computação; Estudos publicados somente na língua inglesa; Estudos publicados desde 2003; Estudos que respondam as questões de pesquisa; Estudos que foquem nas metodologias pedagógicas e/ou computacionais para a educação de surdos; Estudos que tenham tecnologias aplicadas à educação de surdos. Já os critérios de exclusão utilizados foram: Estudos que não são artigos completos (slides Power point, resumo, etc.); Capítulo de Livro; Artigos não escritos em Inglês; Estudos publicados antes de 2003; Não relacionados com o tema: Uma Revisão Sistemática sobre a Educação do Surdo em Ambientes Virtuais Educacionais; Estudos não relacionados com as questões de pesquisa; Estudos duplicados, Estudos sobre educação do surdo sem o uso de tecnologia; Estudos que tenham tecnologia para o surdo fora do contexto da educação; Estudos que abordam outros tipos de deficiência (exceto a surdez); Estudos que apontam soluções genéricas (para várias deficiências); Artigos com tamanho menor de 5 páginas (short papers). Vale ressaltar que, o critério "Estudos publicados somente na língua inglesa" justifica-se 
pelo seu caráter universal, tendo em vista que a maior parte dos trabalhos publicados nas principais bases de dados na área de Computação são escritos na língua inglesa, inclusive artigos brasileiros.

Os critérios de inclusão e exclusão foram usados em todas as etapas do processo de seleção de estudos, sendo que a cada uma dessas etapas, os estudos foram selecionados de acordo com tais critérios, ficando ou não para a próxima fase, como mostrado na Figura 1.

\subsection{Extração dos dados}

Após a realização da busca e seleção de estudos, a extração de dados se deu em forma de leitura total dos artigos selecionados. Com o objetivo de guiar o processo de extração e coleta de dados, o modelo de Kitchenham e Charters (2007) foi adaptado da seguinte maneira. Durante este estágio, foram extraídos 29 estudos primários incluídos na revisão sistemática, de acordo com o formulário de extração (ver Tabela 1). Este formulário contém dados gerais dos artigos, como também dados que ajudam a respondem as questões de pesquisa propostas nesta revisão sistemática. Assim como o processo de seleção, na extração também utilizou-se da ferramenta StArt.

No formulário de extração temos as seguintes informações: identificador, autores, ano de publicação, país, fonte do artigo, referência bibliográfica, número de citações no Google Acadêmico, objetivo do estudo, método de pesquisa e nome da contribuição, assim como critérios específicos de cada questão de pesquisa (Tabela 1).

Tabela 1: Formulário de Extração

\begin{tabular}{|c|c|}
\hline Dados de Estudo & Descrição \\
\hline \multicolumn{2}{|l|}{ ID } \\
\hline \multicolumn{2}{|l|}{ Nome da contribuição } \\
\hline \multicolumn{2}{|l|}{ Data da extração } \\
\hline \multicolumn{2}{|l|}{ Autor } \\
\hline \multicolumn{2}{|l|}{ Ano } \\
\hline \multicolumn{2}{|l|}{ País } \\
\hline \multicolumn{2}{|l|}{ Fonte do artigo } \\
\hline \multicolumn{2}{|l|}{ Referência bibliográfica } \\
\hline \multicolumn{2}{|l|}{ Objetivo do estudo } \\
\hline Método de pesquisa & $\begin{array}{l}\text { (Experimento, Estudo de caso, Survey, Etnografia, Pesquisa-Ação, Cenário } \\
\text { Ilustrativo) }\end{array}$ \\
\hline Maturidade da pesquisa & $\begin{array}{l}\text { (Pesquisa de validação, Pesquisa de avaliação, Proposta de solução, Artigos } \\
\text { filosóficos, Artigos de opinião, Artigos de Experiência) }\end{array}$ \\
\hline Línguas de Sinais & (LIBRAS, ASL, LFS, DSL, etc.) \\
\hline $\begin{array}{l}\text { Atributos relacionados à } Q P 1 \\
\text { Interação do surdo no ambiente onl }\end{array}$ & (GUI, WUI, TUI, NUI, CLI) \\
\hline $\begin{array}{l}\text { Atributos relacionados à } Q P 2 \\
\text { Ambiente online de Aprendizagem } \\
\text { Tecnologia Assistiva }\end{array}$ & $\begin{array}{l}\text { (LMS, CSCL, M-leargning, T-learning, MOOC, ITS), } \\
\text { (tradutor, dicionário, vídeo, escrita de sinais, avatar) }\end{array}$ \\
\hline $\begin{array}{l}\text { Atributos relacionados à } Q P 3 \\
\text { Público alvo }\end{array}$ & (criança surda, criança ouvinte, adulto surdo, adulto ouvinte, indefinido) \\
\hline
\end{tabular}

$\mathrm{Na}$ fase seguinte, os dados coletados serão mostrados em forma de tabelas e gráficos, onde tais dados serão analisados individualmente de acordo com os critérios de extração. Os atributos relacionados especificamente com as questões de pesquisa serão abordados com maiores detalhes a seguir, separando os resultados da análise e discussão de cada questão. 


\section{Resultados e Discussão}

Nesta Seção será feita uma apresentação geral dos estudos retornados através da análise de alguns atributos contidos no formulário de extração (Tabela 3), e logo em seguida os resultados relacionados às questões de pesquisa serão apresentados e discutidos de forma mais detalhada.

\subsection{Visão Geral dos Estudos}

Nesta subseção serão discutidos os resultados da extração com relação aos dados gerais dos estudos, como mostrado no formulário de extração (Tabela 1), onde os mesmos são identificados por um ID, e podem ser acessados em: https://www.dropbox.com/s/4dfmobnywc05803/Systematic\%20Literature\%20Review\% 20References.pdf

\subsubsection{Ano de Publicação:}

Foram analisados estudos no período dos últimos dez anos, ou seja, entre 2003 e 2013. O ano corrente (2014) não foi considerado, pois se encontra em andamento, não sendo adequado obter resultados de um período não concluído. $\mathrm{O}$ ano com maior número de publicações foi 2010 (24,14\%), seguido de 2013 (20,69\%), 2011 (17,24\%), 2007 e 2012 (10,34\%), 2008 e 2009 (6,9\%), 2005 (3,45\%). Nos anos 2003, 2004 e 2006 não tiveram estudos selecionados.

Como observado, o maior número de publicações ocorridas no intervalo predefinido, foi em 2010. Vale ressaltar que apesar de não haver tantos estudos sobre o tema entre os anos de 2003 e 2010, o interesse pela área vem crescendo nos últimos anos, tendo uma boa média entre os anos de 2010 e 2013.

\subsubsection{Método de Pesquisa:}

A classificação dos estudos publicados foram baseados em cinco categorias (Experimento, Estudo de Caso, Survey (questionário), Etnografia, Pesquisa-Ação) definidas por Easterbrook et al (2008). No entanto, a categoria proposta "QuasiExperimento" não foi considerada por sua semelhança com a categoria "Experimento", e também precisou-se de uma nova categoria (Cenário Ilustrativo) para demonstrar estudos que utilizam de pequenos exemplos para avaliar suas contribuições. A maior parte dos estudos utilizaram o método Cenário Ilustrativo $(68,97 \% ; 20$ estudos), seguido de Survey (20,69\%; 6 estudos), Experimento (17,24\%; 5 estudos), Estudo de Caso (3,45\%; 1 estudo), Etnografia (3,45\%; 1 estudo) e Pesquisa Ação (0\%, 0 estudos).

\subsection{Interação do Surdo em Ambientes Virtuais (QP1)}

Esta subseção apresenta os resultados relacionados à QP1, além de discutir as principais implicações de tais resultados.

\subsubsection{Resultados:}

O objetivo desta questão de pesquisa foi identificar quais formas de interação o usuário surdo pode utilizar dentro dos ambientes virtuais de aprendizagem. Foi utilizada a classificação de formas de interação propostas por Bittencourt \& Baranauskas et al (2013) (GUI - Graphical User Interface; WUI - Web User Interface; TUI - Tangible 
User Interface; NUI - Natural User Interface; e CLI - Command Line Interface). Tabela 2 apresenta os resultados encontrados nessa questão de pesquisa.

Tabela 2: Interfaces de Interação

\begin{tabular}{lllc}
\hline Interfaces & Estudos (ID) & Quantidade & \% \\
\hline GUI & E02, E03, E10, E11, E13, E17, E18, E24, E27 & 9 & $31,03 \%$ \\
WUI & E01, E04, E05, E06, E07, E08, E09, E13, E14, E15, E16, E19, & 18 & \\
& E20, E22, E23, E25, E26, E28 & 0 & \\
TUI & & 1 & $0 \%$ \\
NUI & E12 & $07 \%$ & $3,45 \%$ \\
CLI & E29 & 1 & $3 \%$ \\
Não Aplicável & & & $3,45 \%$ \\
\hline
\end{tabular}

\subsubsection{Análise e Discussão:}

Em resumo, a maior parte das interfaces apresentadas foi do tipo WUI $(62,07 \%$; 18 estudos), o que faz bastante sentido, pois as atuais plataformas de ensino funcionam na web. Logo em seguida apareceu a GUI (31,03\%; 9 estudos) onde o ambiente rodava apenas em máquinas locais, e a NUI $(3,45 \%, 1$ estudo), sendo o único estudo que utiliza reconhecimento de voz. A TUI e CLI ( $0 \%$; 0 estudos) não foram utilizadas, e apenas um estudo $(3,45 \%)$ não teve um tipo específico de interface identificado.

\subsection{Tecnologias Assistivas utilizadas em Ambientes Virtuais (QP2)}

Esta subseção apresenta os resultados relacionados à QP2, além de discutir as principais implicações de tais resultados.

\subsubsection{Resultados:}

O objetivo desta questão de pesquisa é levantar quais tecnologias assistivas estão sendo aplicadas aos ambientes virtuais de aprendizagem. Nesse contexto, após discussões dos autores, criou-se uma classificação das principais tecnologias assistivas direcionadas ao surdo, sendo elas: tradutor (traduz da língua escrita para a língua de sinais); dicionário (banco de sinais organizados e conectados às palavras com seus respectivos significados); vídeo (vídeos gravados/filmados de uma pessoa realizando determinado sinal); escrita de sinais ou signwriting (sistema de escrita de línguas gestuais representada por símbolos); e avatar (personagem 2/3D que representa uma pessoa que realiza os sinais). Assim como os principais tipos de ambientes virtuais de aprendizagem existents, sendo eles: LMS - Learning Management System, CSCL Computer Supported Collaborative Learning, M-Learning - Mobile Learning, TLearning - Television Learning, MOOC - Massive Open Online Course e ITS Intelligent Tutoring System. Sendo assim, a combinação entre ambientes virtuais de aprendizagem com alguma tecnologia assistiva, pode caracterizar um ambiente educacional inclusivo, desde que o mesmo consiga atender às necessidades de seus usuários.

Podemos observar na Figura 3, um mapa (do tipo bubble chart) dos dados utilizados na classificação criada, com o objetivo de obter uma visão mais precisa de quais tecnologias assistivas (eixo y) têm sido utilizadas em ambientes virtuais de aprendizagem (eixo $\mathrm{x}$ ), e quais seriam esses ambientes. $\mathrm{O}$ tamanho de cada circunferência (bubble) é definido pelo número de estudos encontrados nas duas categorias. 


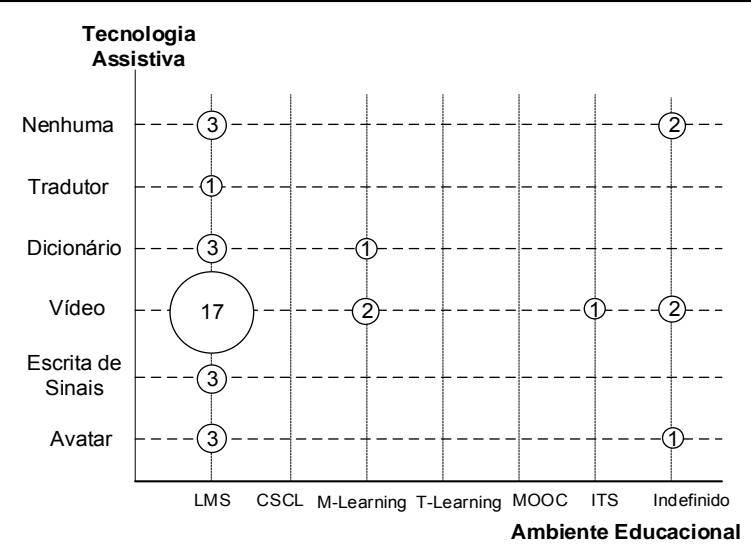

Figura 3: Mapa de distribuição dos estudos por Tecnologia Assistiva e Ambientes Educacionais

\subsubsection{Análise e Discussão:}

Como observado na Figura 3, a maior parte dos estudos que possui algum tipo de tecnologia assistiva utiliza LMS como ambiente virtual, enquanto que alguns tipos de ambientes estão sendo utilizados sem nenhuma associação com quaisquer tecnologias assistivas, como CSCL, T-Learning e MOOCs. Também notamos que com o avanço da tecnologia móvel, alguns recursos de acessibilidade ganharam espaço nas plataformas M-learning (2 estudos), onde em um único estudo encontrado possuía duas modalidades de tecnologia assistiva (vídeo e tradutor).

Quanto à tecnologia assistiva mais utilizada, encontra-se o "Vídeo" (17 estudos), por ser de fácil confecção e inserção nos atuais ambientes virtuais. Seguido de uma minoria com "Avatar" (4 estudos), onde ao contrário do "Vídeo", a confecção de sinais em ambientes de animação 3D não é um processo simples, "Dicionário" (4 estudos) e Escrita de Sinais (3 estudos).

\subsection{Contribuições direcionadas ao modelo de educação inclusiva (QP3)}

Esta subseção apresenta os resultados relacionados à QP3, além de discutir as principais implicações de tais resultados.

\subsubsection{Resultados:}

O objetivo dessa questão de pesquisa é investigar se há soluções direcionadas à educação básica e tem como objetivo proporcionar um modelo de educação inclusiva, modelo este proposto por Fonseca (2004) que defende a ideia de uma educação que ofereça oportunidades educativas equitativas, considerando o acolhimento de pessoas com Necessidades Especiais Educacionais (NEE), no nosso contexto, crianças surdas e ouvintes. Para isso utilizou-se apenas um critério de extração "Público Alvo" (Criança Surda, Criança Ouvinte, Adulto Surdo, Adulto Ouvinte e Indefinido), onde identificamos se a solução é direcionada à crianças em seus primeiros anos escolares. Salvo que no processo de análise, um mesmo estudo pode ser considerado para mais um perfil de público alvo, como observado na Tabela 3.

Tabela 3. Público Alvo

\begin{tabular}{|c|c|c|c|}
\hline $\begin{array}{l}\text { Público Alvo } \\
\end{array}$ & Estudos (ID) & Quantidade & $\%$ \\
\hline Criança Surda & E01, E03, E10, E11, E20, E23, E24, E26, E27 & 9 & $31,03 \%$ \\
\hline Criança Ouvinte & E11 & 1 & $3,45 \%$ \\
\hline Adulto Surdo & $\begin{array}{l}\text { E02, E04, E05, E07, E08, E09, E12, E13, E14, E15, E16, E17, } \\
\text { E19, E21, E22, E25, E28 }\end{array}$ & 17 & $58,62 \%$ \\
\hline Adulto Ouvinte & E06, E18 & 2 & $6,90 \%$ \\
\hline Indefinido & E29 & 1 & $3,45 \%$ \\
\hline
\end{tabular}




\subsubsection{Anlálise e Discussão:}

Em resumo, a Tabela 3 mostra que mais da metade das soluções assistivas atualmente são direcionadas a adultos surdos (58,62\%; 17 estudos), que na maioria das vezes são de caráter imediato, ou seja, não estão preocupados em sanar o problema da exclusão do surdo em seu cerne. Porém uma boa fatia (31,03\%; 9 estudos) está preocupada em promover uma educação direcionada a criança surda. Somente um estudo $(3,45 \%$; ID(E11)) propõe um ambiente focado num modelo que atenda tanto a criança surda, quanto a ouvinte, caracterizando um modelo de educação inclusiva ideal. Dois estudos direcionados para adultos ouvintes $(6,9 \%)$, onde vale ressaltar que um destes $(3,45 \%$; ID(E18)) está direcionado a pais ouvintes que possuem filhos surdos. E por fim, apenas um estudo $(3,45 \%)$ não foi possível identificar o seu público alvo.

\section{Ameaças à Validade}

Para que a execução desta revisão sistemática fosse coerente, o processo de seleção dos estudos utilizou-se de critérios de inclusão e exclusão criandos antes do início da revisão. A busca foi realizada num conjunto das principais bases de dados da área, num intervalo de tempo dos últimos dez anos, sendo possível que estudos relevantes não tenham sido incluídos. Para minimizar essa ameaça, o processo de seleção e os critérios de inclusão e exclusão foram cuidadosamente projetados e discutidos pelos autores para minimizar o risco de exclusão de estudos relevantes.

Outra ameaça à validade identificada pode estar presente em algumas decisões subjetivas ocorridas durante o processo de seleção e extração de alguns estudos que não apresentaram uma descrição clara, dificultando a aplicação objetiva dos critérios de inclusão e exclusão ou a imparcialidade na extração de dados. Para minimizar esta ameaça, o processo de seleção foi feito de forma iterativa e extração de dados foi feita de forma colaborativa pelos revisores, e os conflitos foram discutidas e resolvidas por todos os autores. Na verdade, dois revisores realizaram a seleção em paralelo e os seus resultados de seleção foram harmonizados para minimizar o viés pessoal no resultado de seleção causada pelo processo de revisão individual.

\section{Considerações Finais}

O objetivo deste estudo foi levantar as pesquisas existentes com relação a educação do surdo em ambientes virtuais, proposta como principal questão de pesquisa deste artigo. Para cumprir tal objetivo foi conduzida uma revisão sistemática da literatura.

De acordo com os resultados, a forma de interação mais comum em ambientes virtuais utilizada por surdos para a obtenção de conhecimento é a WUI, com 18 estudos $(62,07 \%)$ respondendo a QP1, já que a maioria dos ambientes estão disponíveis na Web. Dentre as tecnologias assistivas mais utilizadas nesses ambientes, o Vídeo teve grande destaque, aparecendo em 21 estudos $(72,41 \%)$, por ser um meio bastante simples de criação de material, enquanto que o ambiente virtual de aprendizagem mais utilizado foi o LMS, com 22 estudos (75,86\%), e o cruzamento destes apareceu em 17 estudos $(58,62 \%)$, respondendo assim a QP2 . E por fim, percebemos que apesar de haverem 9 estudos $(31,03 \%)$ direcionados a crianças surdas, apenas um estudo (3,45\%) foi considerado como modelo ideal de educação inclusiva, considerando a condição que a tecnologia deve atender crianças surdas e ouvintes simultaneamente, respondendo a QP3. 
Como trabalho futuro desta revisão, deseja-se implementar novas soluções assistivas com o intuito de colaborar com o modelo de educação inclusiva dito neste trabalho.

\section{Referências}

Bersch, R. "Introdução à Tecnologia Assistiva. Assistiva Tecnologia e Educação". Porto Alegre - $\quad$ RS, $2013 . \quad$ Disponível em: http://www.assistiva.com.br/Introducao_Tecnologia_Assistiva.pdf. Acesso em julho de 2014.

Bittencourt, I.; Baranauskas, M. C.; Pereira, R.; Dermeval, D.; Isotani, S.; Jaques, P. “A Systematic Review of Software Tools for Building Multi-device Inclusive Environments". Elsevier Science, 2013.

Brasil. Casa Civil da Presidência da República. LEI N 10.436, DE 24 DE ABRIL DE 2002. Disponível em: http://www.planalto.gov.br/ccivil_03/leis/2002/L10436.htm. Acesso em: julho de 2014.

Easterbrook, S.; Singer, J; Storey, M. A.; Damian, D. "Selecting empirical methods for software engineering research". Guide to Advanced Empirical Software Engineering, F. Shull, J. Singer, and D. Sjberg, Eds. Springer London, 2008, pp. 285-311

Feneis - "Federação Nacional de Educação e Ingegração dos Surdos". WFD - World Federation of the Deaf (Federação Mundial dos Surdos). Disponível em: http://www.feneis.org.br/page/wfd.asp. Acesso em julho de 2014.

Fonseca, V. "Tendências Futuras da Educação Inclusiva”. In: STOBÄUS, C. D; Mosquera, J. J. M. (Orgs). Educação Especial: em direção à educação inclusiva. 2 ed. Porto Alegre: EDIPUCRS, 2004, p. 41-63.

Frias, E. M. A.; Menezes, M. C. B. "Inclusão Escolar do Aluno com Necessidades Educacionais Especiais: contribuições ao professor do Ensino Regular". PDE, FAFIPA, $2008 . \quad$ Disponível em http://www.diaadiaeducacao.pr.gov.br/portals/pde/arquivos/1462-8.pdf. Acesso em julho de 2014.

IBGE. “Censo Demográfico de 2010”. Instituto Brasileiro de Geografia e Estatística. Disponível em: http://www.ibge.gov.br/estadosat/temas.php?sigla=ap\&tema=censodemog2010_defic. Acesso em julho de 2014.

Kitchenham, B.; Charters, S. "Guidelines for performing systematic literature reviews in software engineering". Keele University and Durham University Joint Report, Tech. Rep. EBSE 2007-001, 2007.

Petersen, K.; Feldt, R.; Mujtaba, S.; Mattsson, M. "Systematic mapping studies in software engineering". Proceedings of the 12th International Conference on Evaluation and Assessment in Software Engineering, ser. EASE'08. Swinton, UK, UK: British Computer Society, 2008, pp. 68-77. 